\section{A 90-Degree-Tilt Rotary Adapter for SEM}

James M. Ehrman* and Irena Kaczmarska

Mount Allison University

*Corresponding author, jehrman@mta.ca

Many SEM stages prevent effective examination of specimens at extremely high tilt angles. Stage tilt may not be allowed at or near 90 degrees, and the design of the stage may cause the loss of $X$ or $Y$ stage motion at high tilts. We designed an adapter for our microscope that solves both problems by shifting the normal stage rotation motion 90 degrees (Figure 1). Specimen stubs are mounted horizontally in the adapter, and all components of the holder except the base plate are tethered to a stationary portion of the stage. Operation of the stage rotation control then rotates the stub around its horizontal axis (similar to a rotisserie). Specimens can be rotated through 360 degrees along this axis, with a full range of $X$ and $Y$ stage movement. $A$ limited amount of stage tilt is also possible, allowing precise positioning of the specimen.

Using the adapter has several advantages. First, all sides of a specimen, with the exception of the mounting side, can be examined (Figure 2A-C). The adapter is particularly useful for examination of insects and other small organisms. If the specimen is mounted laterally on a supporting pin, it can be imaged from a true lateral view (in the regular stub holder) and then transferred to the rotary adapter for anterior, posterior, dorsal and ventral views, as well as any orientation in between. This capability can be invaluable when examining extremely rare specimens, in instances where only one specimen is available, or cases where all images must come from the same specimen (such as a taxonomic holotype). Without the rotary adapter, only views for which the specimen was originally mounted can be viewed "straight on" in a microscope that does not provide unrestricted tilt (Figure 2D).

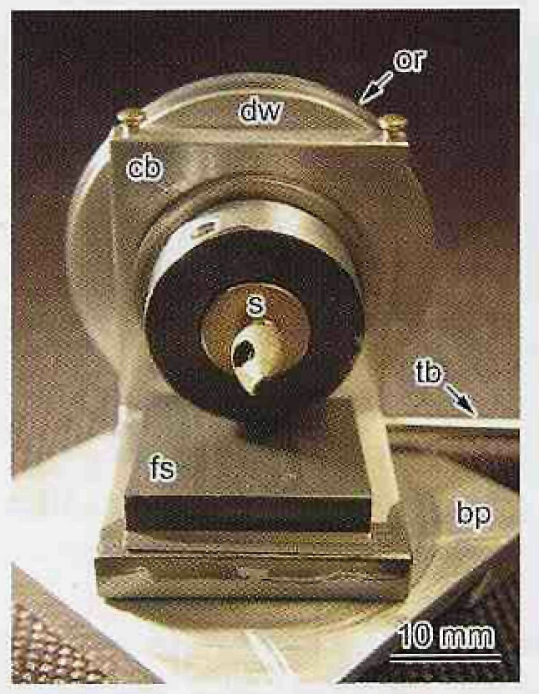

Figure 1: Rotary adapter with SEM specimen stub (s) mounted horizontally. All parts of the adapter except the base plate (bp) are attached to a stationary portion of the stage via a tether bar (tb) extending from the corner of the connecting bracket (cb). Operation of the stage rotation control revolves the specimen on its horizontal axis by transferring stage rotation motion to the drive wheel (dw). An O-ring (or) provides traction between the drive wheel and the base plate. The foil shield ( $f s$ ) painted with carbon paint provides a uniformly dark background in images acquired using the adapter. See http://www.mta.ca/-jehrman/ rotary.htm for a detailed schematic of the adapter.
Another benefit of the rotary adapter is that manipulation of the specimen about the new axis of rotation does not change the orientation of the adapter components beneath the specimen. With the addition of a simple metal foil shield painted with colloidal carbon or similar low secondary electron yield coating (Figure 1), images can be produced with a uniformly dark, featureless background (Figure 2A-C). Similar images produced at high tilt with an ordinary stub holder often contain distracting, overly bright background clutter (Figure 2D). Undesirable backgrounds can be removed from digital images with editing software, but is very timeconsuming for specimens with complex boundaries, and may raise concerns regarding the ethics of extensive digital manipulation of scientific images.

Our design is specific to JEOL " $T$ " series microscopes, but could be easily modified for other instruments. Critical elements and caveats for modifying and using such an adapter include:

1. The height of the adapter should be as small as possible so that it fits into the specimen chamber and provides a long working distance for maximum depth of field on large specimens. Our design provides a working distance of approximately $35 \mathrm{~mm}$ at a stage $Z$ height of $48 \mathrm{~mm}$, depending on the position of the specimen on the stub.

2. The connecting bracket holding the drive wheel to the base plate must be tethered to a stationary component of the stage so that rotation of the stage control is translated to the drive wheel. We fasten the tether bar to a stage support with a nylon electrical cable tie.

3. The base plate should be centered over the axis of stage rotation to prevent lateral drift of the specimen as the specimen is rotated.

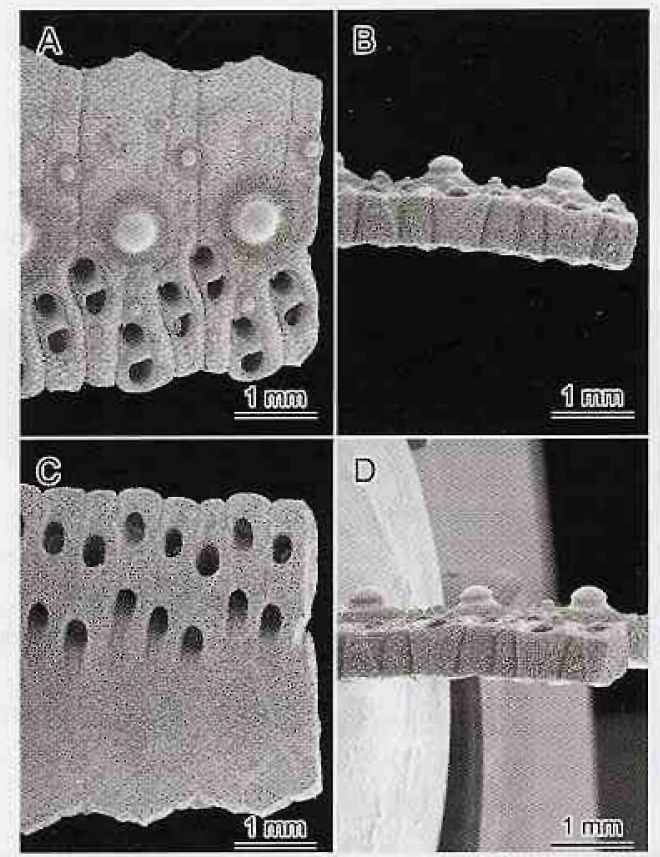

Figure 2: Fragment of a sea urchin endoskeleton, mounted to the SEM stub on-edge. The rotary adapter allows viewing the specimen from any angle (A-C) with a uniformly dark, featureless background. Examination at high tilt with the standard specimen holder (D) often restricts stage movement in the $X$ or $Y$ direction, and can produce distracting background clutter. See http://www.mta.ca/ -jehrman/rotary.htm for an animation of the sea urchin endoskeleton rotated through 360 degrees.

Continued on page 26 


\section{ITO-GOLD SCINTILLATORS}

Give your SEM the best...

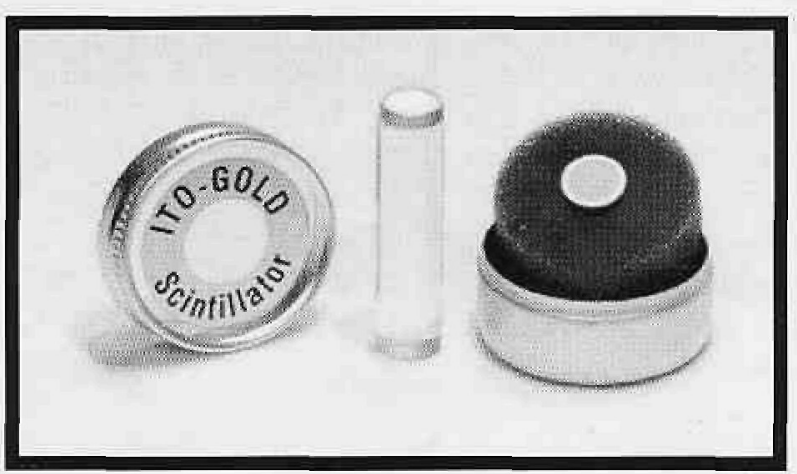

...and get it back in image quality!

Features a gold plated retaining ring, bonded to substrate coated with conductive, transparent indium tin oxide

High quality $P-47$

No aluminum overcoating required

Can be recoated

Provides better electrical contact

Better signal to noise ratio

Conductive substrate reduces "pinhole" interference

Easier to handle during installation

Available for most SEMs

Patent applied for

For more details on this great new product contact:

M. E. Taylor Engineering, Inc.

21604 Gentry Lane, Brookeville, MD 20833

Phone: 301-774-6246 Fax: 301-774-6711

Visit us on the web at:

www.semsupplies.com

Available worldwide! 


\section{A 90-Degree-Tilt Rotary Adapter for SEM Continued from page 24}

4. The fit between components should be tight enough so that traction provided by the O-ring turns the drive wheel, but not so tight as to produce binding. Mating surfaces should be polished smooth and lubricated with a small amount of vacuum grease.

5. The bottom of the base plate should be machined so that it attaches to the stage in a similar fashion as standard specimen holders.

6. Components below the specimen (machine screw and washer) should be masked with a foil shield coated with carbon paint to provide a uniformly dark background.

7. As with any large specimen and/or holder, the rotary adapter should be used with caution. Care should be taken especially with manipulation of stage height and tilt controls, to prevent collision damage with other components in the column.

\section{Acknowledgments}

We are routinely grateful for the patience and skill of $\mathrm{Al}$ MacDonald, the machinist wizard who crafted this device and many others in our lab. This adapter was modeled after a less elaborate device designed by James M. Ehrman and J.R. Scott at the Texas A\&M University Electron Microscopy Center in 1981. The Natural Sciences and Engineering Research Council of Canada (NSERC), the Canada Foundation for Innovation (CFI), and Mount Allison University have provided major funding for the Digital Microscopy Facility.

\section{"The Evactron device can significantly reduce contamination in the SEM."}

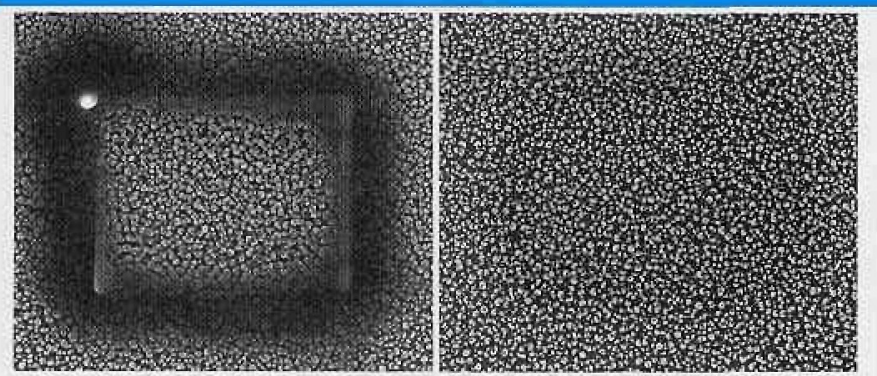

A silicon "grass" sample irradiated for 10 minutes before (left) and after (right) the use of Evactron SEM-CLEAN device. $50 \mathrm{kX}$ From Active Monitoring and Control of Electron Beam Induced Contamination by A. Vladar, M. Postek, \& R. Vane, SPIE Microlithography Conference. Feb. 2001.

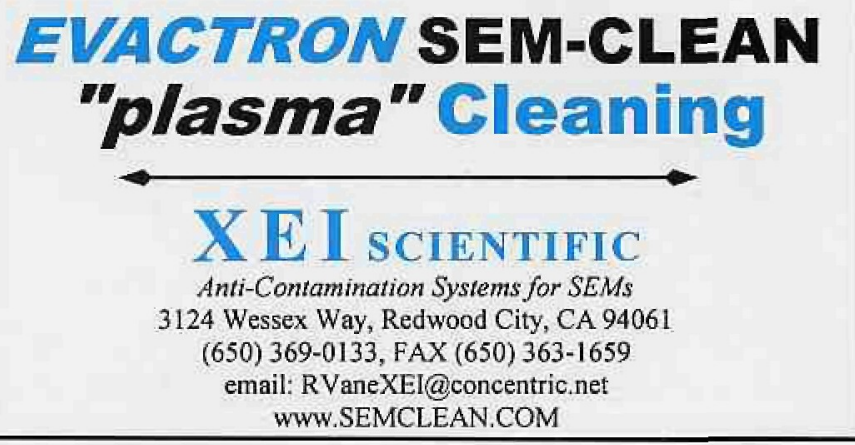

\section{Measuring Conductivity With Scanning Probe Microscopes}

Sergei Kalinin, University of Pennsylvania sergei2@seas.upenn.edu

There are two kinds of conductivity measurements possible with scanning probe microscopy (SPM). In the first case, the specific resistance of material directly below the tip is probed. In the second case, SPM probes local potential induced by the lateral current applied through macroscopic contacts, thus providing the information on the mesoscopic transport properties of the sample.

The first set of techniques is invariably based on measuring tip-surface current in contact or intermittent tapping mode. If the tip-surface contact resistance is small (good contact), the current will be limited by the spreading resistance of the sample from which specific resistance can be calculated, assuming that the contact area is known. In practice, good tip-surface contact requires high indentation forces and extremely clean surfaces, especially for semiconducting oxides. On semiconductor surfaces, space charge layers and Schottky regions below the tip will also affect the measurements. An interesting development of this approach is thin-film measurements, in which case regions with high current density (e.g., defects) can be detected.

The second type of conductivity measurement are based on using potential-sensitive SPM techniques such as scanning surface potential microscopy (SSPM, or Kelvin Probe Force Microscopy, KPFM) on laterally biased surfaces. This setup is very similar to the usual four point resistivity measurements, but instead of two fixed voltage electrodes, the SPM tip acts as a single moving voltage electrode.

For quasi-one dimensional systems such as a metalsemiconductor interface or a grain boundary in a bicrystal, the subsequent analysis is straightforward. Assuming that the sample is connected in series with current limiting resistors of total resistance $R_{t}$ the current is $I=V_{\text {lat }} /\left(R+R_{d}\right)$, where $V_{\text {lat }}$ is lateral bias applied by an external voltage source and $R_{d}$ is voltage dependent interface resistance. The current voltage characteristic of the interface is then $I_{d}\left(V_{d}\right)=\left(V_{\text {lat }}-V_{d}\right) / R$, where $V_{d}$ is potential drop at the interface measured directly by SSPM. The presence of stray resistances in the circuit (e.g., due to the bulk of the sample) can be determined and quantified by varying the current limiting resistor $\mathrm{R}$. Alternatively, the current in the circuit can be measured directly. Such measurements can be conveniently done by applying a slow (approximately milliHz range) triangular voltage ramp across the interface with the slow scan disengaged. The first image is then the SSPM image in which each line corresponds to different lateral bias conditions (i.e., potential profile across the interface, from which $V_{d}\left(V_{\text {lat }}\right)$ is obtained). The second image stores the actual lateral bias $\left(V_{\text {lat }}\right)$ and the third image is current in the circuit measured by an $\mathrm{I}$ - $\mathrm{V}$ converter $\left(\mathrm{I}=\mathrm{I}_{\mathrm{d}}\right)$. A similar approach can be extended to systems with multiple interfaces, such as $p-i-n$ diodes, etc. In all cases, the potential we are interested in is the difference between the potential under bias and the potential of the grounded surface, which takes care of the contact potential difference (CPD) variations across the surface.

Analysis of conductivity in laterally inhomogeneous systems is less straightforward. Qualitatively, detection of resistive barriers (e.g., grain boundaries) is still straightforward. On applying the lateral bias, potential drops develop on electroactive interfaces and can be readily visualized by SSPM. However, the quantitative image analysis in this case is difficult.

One of the factors that has to be taken into account in SSPM 